\title{
Kinetics of ATP release following compression injury of a peripheral nerve trunk
}

\author{
P. Grafe $\cdot$ V. Schaffer $\cdot$ F. Rucker
}

Received: 7 October 2005 / Revised: 19 December 2005 / Accepted: 20 December 2005 / Published online: 11 July 2005

(C) Springer Science + Business Media B.V. 2006

\begin{abstract}
Compression and/or contusion of a peripheral nerve trunk can result in painful sensations. It is possible that release of ATP into the extracellular space may contribute to this symptom. In the present study, we used real-time measurements of ATP-induced bioluminescence together with electrophysiological recordings of compound action potentials to follow changes in the extracellular ATP concentration of isolated rat spinal roots exposed to mechanical stimuli. Nerve compression for about $8 \mathrm{~s}$ resulted in an immediate release of ATP into the extracellular space and in a decrease in the amplitude of compound action potentials. On average, a rise in ATP to $60 \mathrm{nM}$ was observed when nerve compression blocked $50 \%$ of the myelinated axons. After the compression, the extracellular concentration of ATP returned to the resting level within a few minutes. The importance of ecto-nucleotidases for the recovery period was determined by exposure of isolated spinal roots to high concentrations of ATP and by use of inhibitors of ecto-nucleotidases. It was observed that spinal roots have a high capacity for ATP hydrolysis which is only partially blocked by $\beta \gamma$-methylene ATP and ARL 67156 . In conclusion, acute nerve compression produces an increase in the extracellular concentration of ATP and of its metabolites which may be sufficient for activation of purinergic $\mathrm{P} 2$ and/or P1 receptors on axons of nociceptive afferent neurons.
\end{abstract}

Key words ectonucleotidase - luciferase - neuropathic pain . $\mathrm{P} 1$ receptor $\mathrm{P} 2$ receptor

\footnotetext{
P. Grafe $(\bowtie) \cdot$ V. Schaffer $\cdot$ F. Rucker

Department of Physiology, University of Munich,

Pettenkoferstr. 12,

80336 Munich, Germany

e-mail: P.Grafe@lrz.uni-muenchen.de
}

\author{
Abbreviations \\ ARL 67156 6-N,N-Diethyl- $\beta$ - $\gamma$-dibromomethylene-D- \\ adenosine-5-triphosphate \\ $\beta \gamma$-meATP Adenylylmethylenediphosphonate disodium \\ salt \\ CAP Compound action potential \\ eNPP Ecto-nucleotide pyrophosphatase/phospho- \\ diesterase \\ eNTPDase Ecto-nucleoside triphosphate diphosphohy- \\ drolase \\ HEPES N-(2-Hydroxyethyl)piperazine-N'-(2-ethane- \\ sulfonic acid)
}

\section{Introduction}

Mechanical stimuli such as acute contusion or compression of a peripheral nerve trunk are often accompanied by painful sensations [1]. The effects of compression injury on action potential conduction and morphological aspects of axons in bundles of nerve fibers have been studied in detail $[2,3]$ and provide the basis for an explanation of the loss of axonal functions. However, the development of painful sensations, i.e., an abnormal increase in axonal excitability, is much less understood. A factor possibly involved in the transduction of mechanical stimuli into enhanced excitability of nociceptive nerve fibers in the trunk of a peripheral nerve is extracellular ATP and/or its metabolites. It is well documented that ATP is released by different types of mechanical stimuli [4]. Receptors for ATP on nociceptive neurons have also been described [5, 6]. In addition, there is increasing evidence for the presence of ionotropic (P2X) and metabotropic (P2Y) purinergic receptors in the axonal membrane of sensory nerve fibers including slow conduct- 
ing axons in dorsal spinal roots $[7,8]$ and in the membrane of surrounding nonmyelinating Schwann cells, respectively [9-12]. However, there is little quantitative knowledge about the release of ATP in the trunk of a peripheral nerve in response to mechanical stimuli.

After release into the extracellular space, ATP is rapidly hydrolyzed by ecto-nucleotidases. The currently known ecto-nucleotidases include members of the eNTPDase family, eNPP family, alkaline phosphatases, and ecto-5' nucleotidase $[13,14]$. Degradation of ATP in the trunk of peripheral (vagus) nerve has been studied by use of radio active $\left[{ }^{3} \mathrm{H}\right]$ ATP and rapid hydrolysis to ADP, AMP, adenosine, and inosine was found [15]. Recently, by means of enzyme histochemistry and immunostaining, an association of eNTPDase 2 with nonmyelinating Schwann cells of the rat sciatic nerve has been described [16]. It is likely, therefore, that compression of a peripheral nerve trunk will also result in extracellular accumulation of adenosine. A subtype of adenosine receptor (A2) on peripheral terminals and axons of primary nociceptive afferents has been discussed as a possible mediator in the development of neuropathic pain $[5,17,18]$.

To our knowledge, the importance of ecto-nucleotidases for the kinetics of ATP release induced by nerve compression has not been studied before. Extracellular concentrations of ATP can be followed in real time using bioluminescence produced by the luciferin/luciferase reaction [19-21]. In the present study, we used this method for the determination of changes in the extracellular concentration of ATP during and after compression of isolated rat spinal roots. The data demonstrate that nerve compression produces release of ATP with an initial transient peak and a longer lasting recovery period. Activity of ecto-nucleotidases limits the extracellular accumulation of ATP during the compression period and contributes to the kinetics of the following decline in the concentration of ATP. Our data also indicate that receptors for ATP and its metabolites on sensory axons may contribute to the development of painful sensations which accompany compression of peripheral nerve trunks.

\section{Materials and methods}

Animals and preparation

Adult Wistar rats, weighing 250-350 g, were killed by asphyxiation in a chamber filled with carbon dioxide gas. A laminectomy was performed to expose the cauda equina and the spinal ganglia. Spinal roots were removed in their entire length (from the spinal cord to the spinal nerve) for in vitro studies. Spinal roots were separated from the spinal ganglia and stored for up to $48 \mathrm{~h}$ in a HEPES-buffered solution at $4-7^{\circ} \mathrm{C}$.

Recording chambers

Two different types of recording chamber were used for the determination of extracellular ATP concentrations. ATP release by mechanical stimulation was tested in a perspex organ bath in which a single spinal root was held at each end by suction electrodes (distance approximately $3 \mathrm{~mm}$ ). These suction electrodes were used for electrophysiological measurements. Kinetics of ATP hydrolysis were observed by placing four spinal roots in each well of a four-well culture dish (Nunc, Wiesbaden, Germany). The bathing solution in both types of recording chamber consisted of $400 \mu \mathrm{l}$ of a HEPESbuffered saline and $50 \mu \mathrm{l}$ of an ATP assay mix.

\section{Bioluminescence setup}

Experiments were performed using a Zeiss Axiovert 200 microscope in combination with a photon counting unit (Hamamatsu H7421 head and C8855 counting unit). Bioluminescence was recorded in the organ baths placed on the stage of the microscope $(\times 10$ objective; field of view: $2 \mathrm{~mm}$ ). The organ baths used were either equipped with suction electrodes for additional electrophysiological recordings (fixed position for mechanical stimuli, see Figure 2) or connected to a shaker (Variomag teleshake) for continuous recordings of bioluminescence when ATP or other compounds were added to the bathing solution. The bioluminescence setup was placed in a dark room; remote control of the micromanipulator and data acquisition were carried out in the neighboring room using Labview software provided by Hamamatsu. Photon counting was performed with a time resolution of $1 \mathrm{~s}$.

\section{Electrophysiology}

The isolated nerve preparations were held at each end by suction electrodes in an organ bath. One suction electrode was used to elicit action potentials, while the other was used as a recording electrode. The spinal roots were stimulated with a linear stimulus isolator (A395, WPI, Sarasota, FL, USA) with a maximal output of $500 \mu$ A. The stimulator was controlled by a computer via a data acquisition board (Data Translation DT2812, Marlboro, MA, USA) and the software QTRAC (Prof. H. Bostock, Institute of Neurology, London). Maximal compound action potentials of A fibers were tested with $0.1 \mathrm{~ms}$ current pulses at a constant frequency of $1 \mathrm{~Hz}$. 

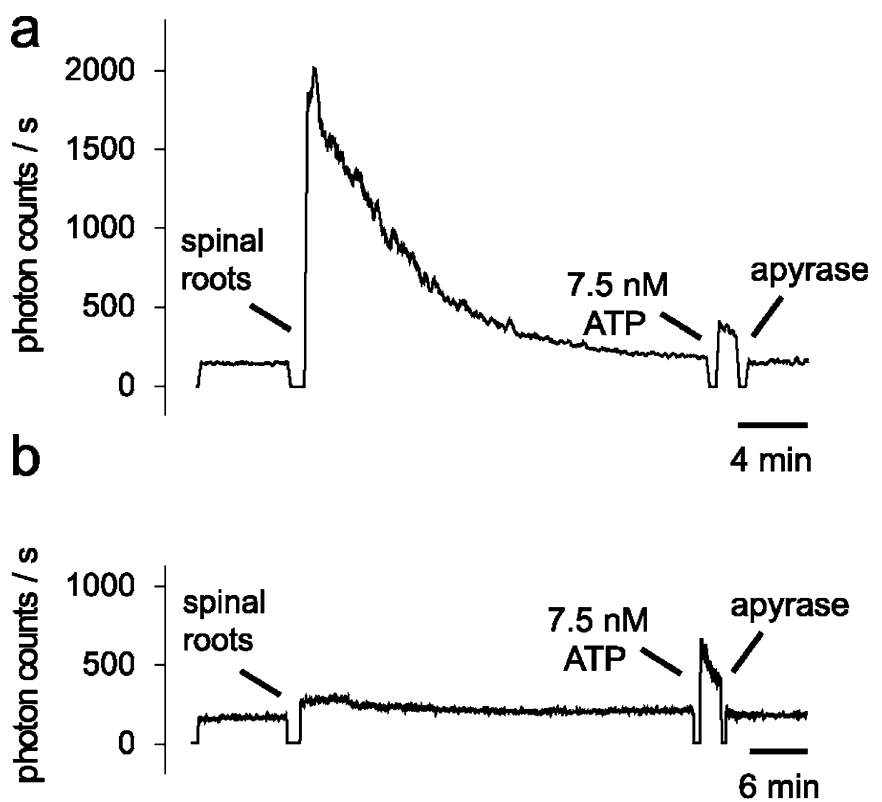

Figure 1 Baseline level of ATP in the recording chamber. a, b Four isolated rat spinal roots were added to the recording chamber filled with $400 \mu \mathrm{l}$ of a HEPES-buffered saline and $50 \mu \mathrm{l}$ of the ATP assay mix (arrow). The spinal roots that were recorded in (a) were squeezed at their endings with tweezers; spinal roots in (b) were transported to the organ bath by means of curved tipped forceps. Note that less mechanical stress induces less luminescence (lower concentration of ATP) in the bathing medium. In either condition, luminescence returns to the baseline level within $10-20 \mathrm{~min}$. Addition of apyrase $(50 \mu \mathrm{g} / \mathrm{ml})$ at

\section{Mechanical stimulation}

The method used for compression of isolated spinal roots is similar to a setup described by Shi and Blight [2]. In the present study, a rod was attached to a motorized micromanipulator (LN-Mini 25, Luigs \& Neumann, Ratingen, Germany). This compression rod was positioned perpendicular to the tissue and was brought to a point of contact with the surface of an isolated spinal root (Figure 2). The rod consisted of two parts, the lower part was free to move against the upper part. The end of the rod had a diameter of $1 \mathrm{~mm}$; the width of a thick dorsal spinal root is about $0.8 \mathrm{~mm}$. The pressure was adjusted by the weight of the movable part $(66 \mathrm{mN})$; the depth of nerve compression was attuned to $0.1-1 \mathrm{~mm}$ by the micromanipulator. The rod was advanced slowly by means of the manipulator motor (about $5 \mathrm{~s}$ ), halted at its lowest position for $3 \mathrm{~s}$, and moved upward afterwards to the home position.

\section{Solutions}

Experiments were performed at room temperature. The organ baths were filled with $400 \mu \mathrm{l}$ of a HEPES-buffered saline and $50 \mu \mathrm{l}$ of a diluted ATP assay mix. The HEPESbuffered solution contained (in $\mathrm{mM}$ ): $\mathrm{NaCl} 118$, Na-

\section{C}

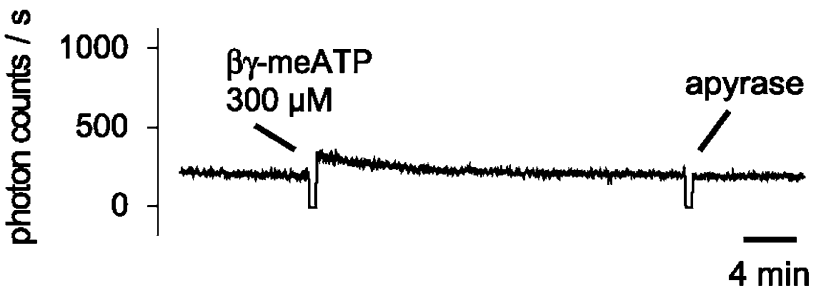

d

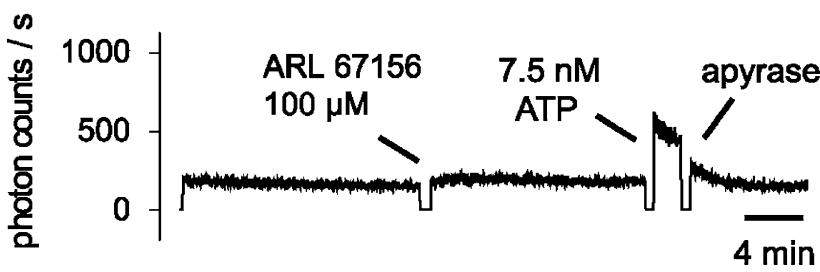

the end of the recording did not lower the number of photon counts/s. This indicates that the baseline level of ATP at this time is at or below the detection limit for ATP.(c, d) Possible contamination of $\beta \gamma$-meATP and ARL 67156 with ATP was tested in bathing media containing spinal roots. Both compounds produced only a small increase in the baseline luminescence which returned to the resting level within a few minutes (for discussion see text). The recording of luminescence in all experiments was temporarily halted when spinal roots or chemicals were added to the recording chamber

gluconate $20, \mathrm{KCl} 3.0, \mathrm{CaCl}_{2} 1.5, \mathrm{MgCl}_{2}$ 1.0, D-glucose 5.0, HEPES 6, and $\mathrm{pH}$ was adjusted to 7.4. For the determination of ATP-induced bioluminescence, one vial of ATP assay mix (FL-AAM, Sigma, Deisenhofen, Germany) was dissolved in $5 \mathrm{ml}$ of sterile water. Aliquots with $100 \mu \mathrm{l}$ were prepared and stored at $-20^{\circ} \mathrm{C} ; 50 \mu \mathrm{l}$ of these aliquots were mixed with $400 \mu \mathrm{l}$ of the HEPES-buffered saline. ARL 67156 and $\beta \gamma$-meATP were purchased from Sigma (Deisenhofen, Germany). A small increase in luminescence was seen when ARL 67156 and $\beta \gamma$-meATP were added to the bathing medium (saline and ATP assay mix). However, this increase in luminescence recovered to the resting level within a few minutes (Figure 1c and 1d). The most likely interpretation is that the spinal roots degrade contaminating ATP. Therefore, ARL 67156 and $\beta \gamma$-meATP were added to the bathing medium at least $15 \mathrm{~min}$ before the experimental test (addition of ATP or nerve compression).

Calibration curves for ATP-induced bioluminescence were determined by addition of ATP $(15$ and $150 \mathrm{nM})$ into the bathing solution at the end of the experiments (see Figure 7). A two-point calibration is sufficient because there is a linear relationship between ATP concentrations and the light intensity (measured as photon counts/s) produced by the assay mix in this concentration range of ATP (e.g., [22]). Higher concentrations of ATP were not tested, 


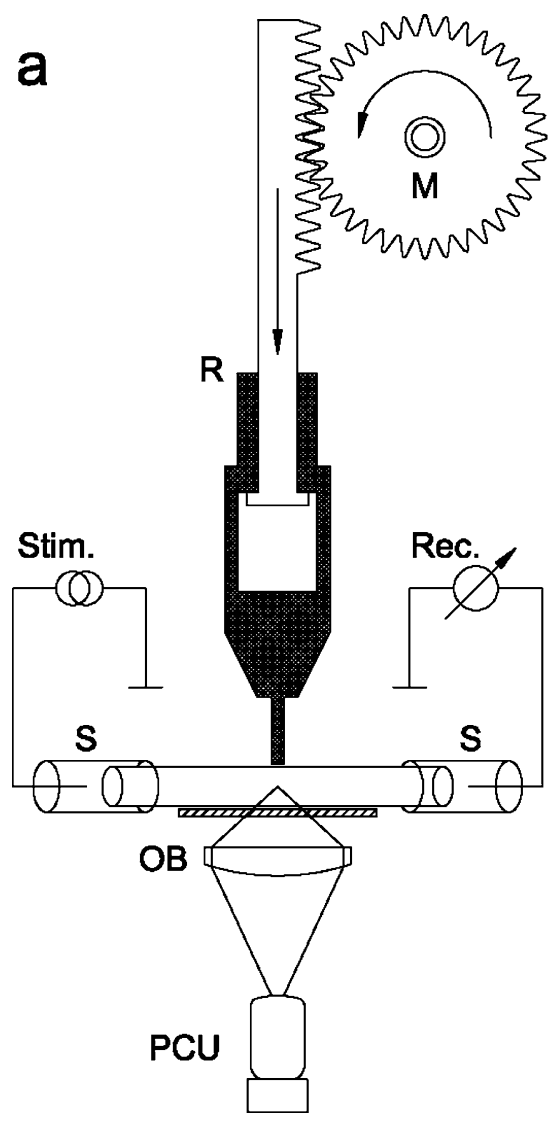

Figure 2 Schematic drawing of the method applied for detection of ATP release during compression of isolated spinal roots. (a) A compression rod attached to a motorized micromanipulator was positioned perpendicular to the tissue and was brought to a point above the surface of an isolated spinal root. The rod consisted of two parts, the lower part was free to move against the upper part. The end of the rod had a diameter of $1 \mathrm{~mm}$; the width of a thick dorsal spinal root is about $0.8 \mathrm{~mm}$. The rod was advanced slowly by means of the manipulator motor. The bath solution contained an ATP assay mix;

because light emission with flash kinetics is produced by firefly luciferase and ATP concentrations $>1 \mu \mathrm{M}$ [23].

\section{Statistics}

Data are expressed as mean \pm SEM. Statistical analysis was performed by an unpaired two-tailed $t$-test to assess significance of differences.

\section{Results}

\section{Baseline level of ATP}

In initial experiments, the contribution of ATP to the background light level obtained in the bathing solution with added spinal roots was determined. An apparent light intensity of 140-180 photon counts/s was measured by the photomultiplier with a shut optical pathway (dark current). The number of photon counts/s increased up to 500 when bioluminescence was detected by the photon counting unit (PCU). In addition, suction electrodes at both ends of the isolated spinal roots were used for stimulation and recording of compound action potentials. (b) Illustration of the cycle of nerve compression: the rod moved from the home position above the spinal root to the tissue surface, reached its lowest position within $5 \mathrm{~s}$, halted at this position for $3 \mathrm{~s}$, and moved upward afterwards to the home position. The total time of the compression period was about $8 \mathrm{~s}$

the photomultiplier was in contact with the organ bath containing saline and the ATP assay mix. The most important component in this background light level is the residual light emission in the darkened laboratory room. Addition of spinal roots to the organ bath produced additional luminescence which differed quantitatively depending on the mechanical stress during the preparation period. Two typical examples are illustrated in Figure 1. Spinal roots with squeezed endings (tweezers) increased the luminescence to several thousand photon counts/s (Figure 1a). In contrast, the luminescence changed only slightly when the spinal roots were transported and added to the organ bath by means of curved tipped forceps (Figure 1b). However, in either situation, luminescence returned to the baseline level with about 10-20 min. Furthermore, no further decrease in the number of photon counts was observed after addition of apyrase, an enzyme that catalyzes the hydrolysis of ATP. This indicates that at this point in time the baseline level of ATP was at or below 


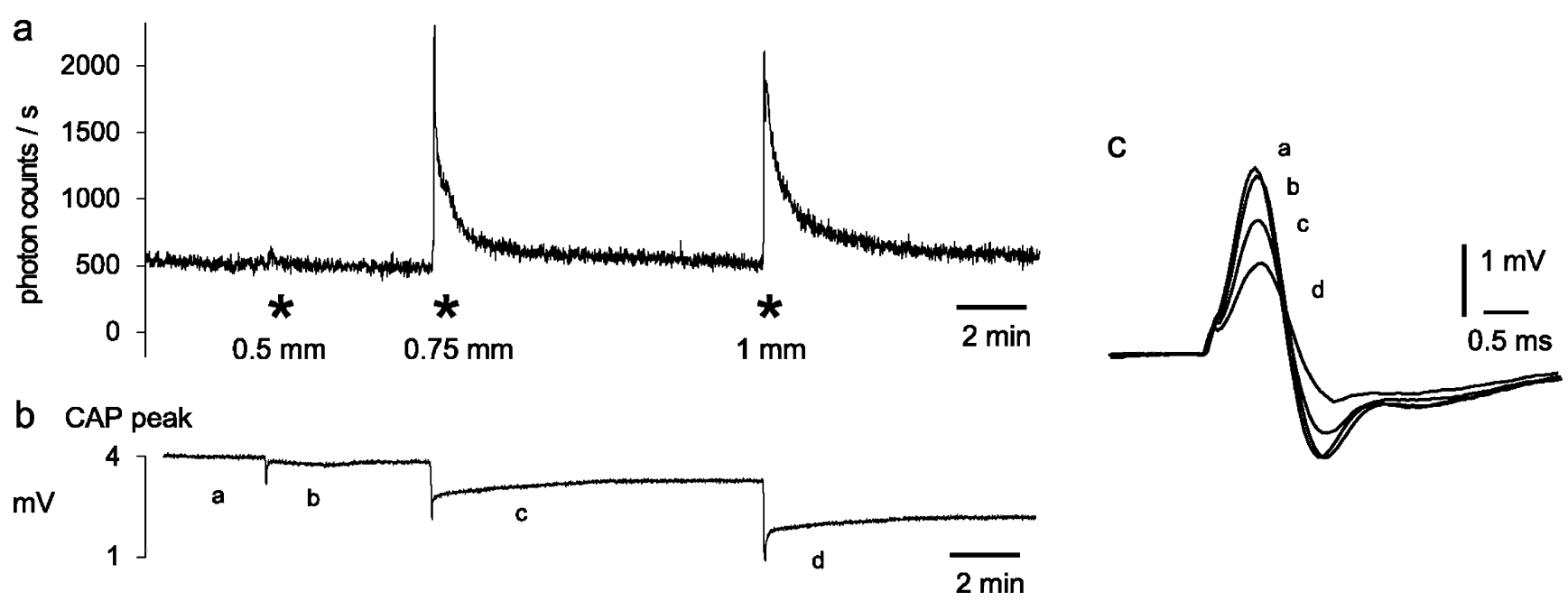

Figure 3 Mechanical stimuli release ATP from an isolated rat spinal root. The isolated spinal root was fixed by two suction electrodes in an organ bath which contained a mixture of saline and an ATP assay mix. Conduction of action potentials by myelinated nerve fibers was tested by recording of the A fiber compound action potential (CAP). (a) The compression rod (see Figure 2) was transiently advanced from the surface of the nerve bundle to a depth between 0.5 and $1 \mathrm{~mm}$ for about $8 \mathrm{~s}$ at the time points indicated by asterisks. Release of ATP due to

the detection limit of the ATP assay mix (around $1 \mathrm{nmol} / \mathrm{l}$ ). Based on this observation, we waited in all experiments for at least 20 min before a test (nerve compression or addition of ATP) was performed.

\section{Transient nerve compression releases ATP}

The combination of continuous photon counting, electrophysiological recording of compound action potentials, and remote control of a micromanipulator was used to study the effects of mechanical stimuli on extracellular ATP concentration and conduction of action potentials in myelinated axons of isolated spinal roots with high time resolution. A typical example of such experiments is illustrated in Figure 3. A rod with a tip diameter of $1 \mathrm{~mm}$ was placed on the surface of an isolated rat dorsal spinal root and was used for transient compression of the nerve trunk with various compression depths. The periods of compression produced transient elevations in the extracelluar concentration of ATP (measured as an increase in photon counts/s) and changes in the compound action potential (CAP) of myelinated A fibers. There was an immediate transient decrease in the peak of the CAP followed by a long lasting partial loss of conducting A fibers.

For the quantitative analysis, release of ATP and the concomitant changes in electrophysiological parameters were studied in 18 spinal roots (Figure 4). There was a clear correlation between the percentage of decrease in the peak height of the CAP and the rise in extracellular ATP. On average, nerve compression sufficient to block conduc- compression of the nerve fibers was detected by the generation of bioluminescence in the solution surrounding the spinal root (measured as photon counts/s). (b) Continuous registration of the peak amplitude of A fiber CAPs. Note, that nerve compression produces an initial transient decrease in the peak amplitude, followed by a long lasting period with partial recovery. (c) Illustration of superimposed examples of CAPs recorded at the points of time marked by $a-d$ in registration (b)

tion in $50 \%$ of the myelinated A fibers resulted in an increase of the ATP concentration in the organ bath surrounding the spinal root by about $60 \mathrm{nM}$.

Fast kinetics of ATP degradation in peripheral nerve trunk

Compression-induced release of ATP produced a transient elevation of the extracellular ATP concentration (Figure 3). Three different factors may contribute to the kinetics of this

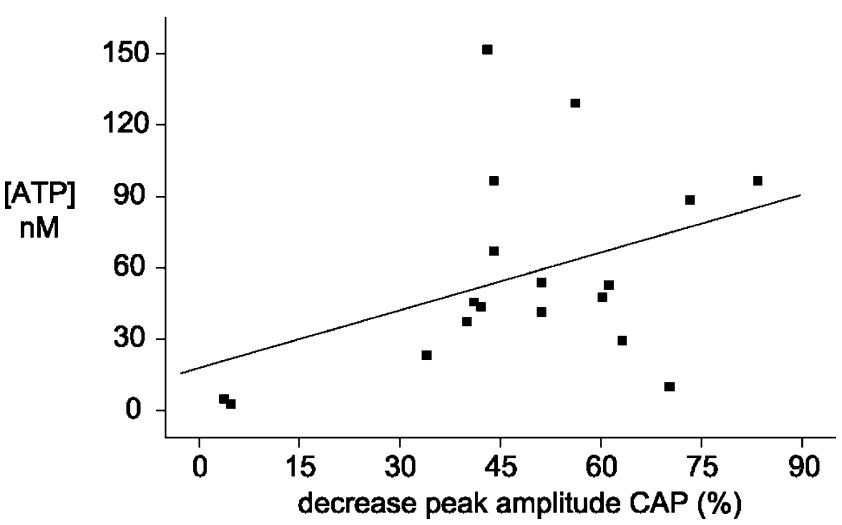

Figure 4 Relationship between release of ATP and electrophysiological changes produced by compression of isolated spinal roots. Summary of data obtained from experiments on 18 spinal roots exposed to nerve compression similar to the experimental protocol illustrated in Figure 3. The decrease in the amplitude of the A fiber compound action potential (CAP) measured $1 \mathrm{~min}$ after nerve compression is plotted against the rise in the extracellular concentration of ATP (measured in the solution surrounding the isolated spinal root at about $15 \mathrm{~s}$ after nerve compression). The regression line indicates a correlation between the release of ATP and the extent of decrease in axonal excitability 

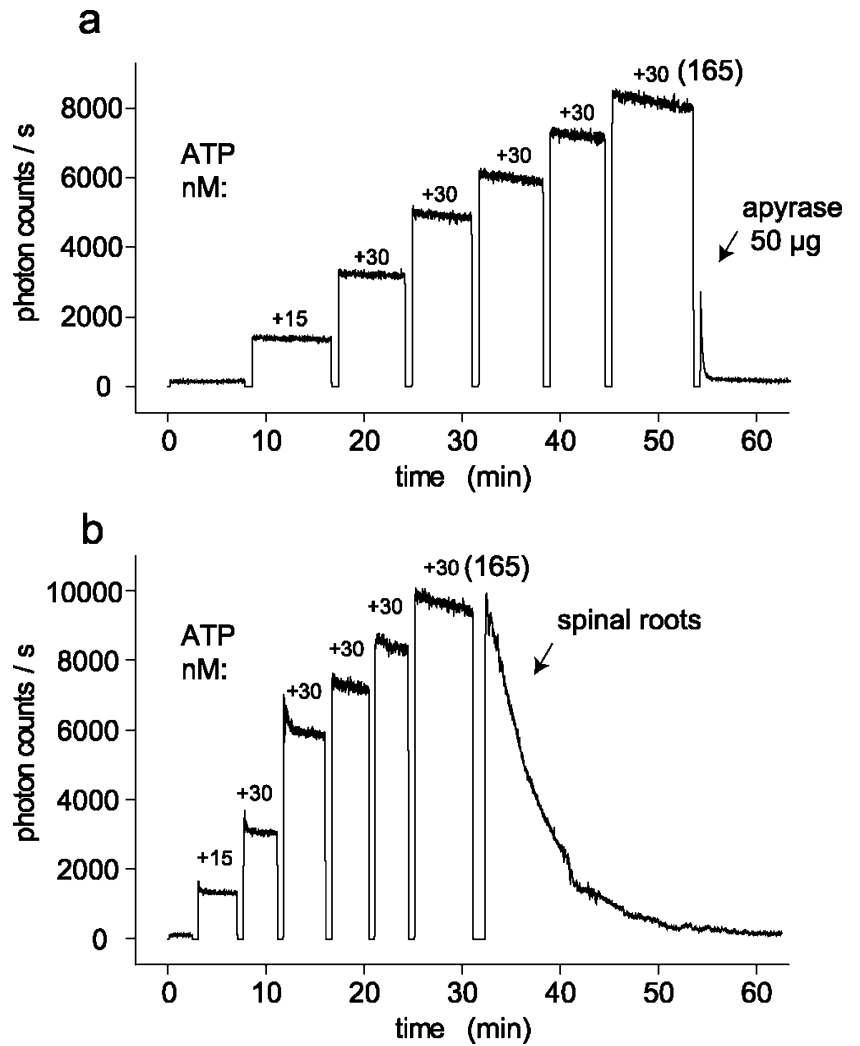

Figure 5 Spinal roots rapidly degrade extracellular ATP. (a) ATP was added successively to a rotating culture dish (containing saline and the ATP assay mix) until a final concentration of $165 \mathrm{nM}$ ATP was reached. Afterwards, apyrase was added and blocked the ATP-induced bioluminescence by hydrolysis of ATP (the photomultiplier was shut down during addition of solutions to the culture dish; note discontinuity in the recording of bioluminescence). (b) In this experiment, after an ATP concentration of $165 \mathrm{nM}$ was reached, four isolated rat spinal roots were added to the culture dish. Note the rapid decline in the bioluminescence which indicates degradation of ATP by the nervous tissue. In contrast, consumption of ATP by the enzymatic reaction of the ATP assay mix is much slower

decline: (1) diffusion of ATP from the surface of the isolated spinal roots into the organ bath, (2) consumption of ATP by the light-producing enzymatic reaction, and (3) degradation of ATP by ecto-nucleotidases within the nerve trunk [19]. Several experiments were performed with the aim of separating these possible factors. First, the ability of isolated spinal roots to hydrolyze extracellular ATP was tested in culture dishes mounted in connection with a shaker on the stage of the microscope. A typical experiment which demonstrates fast degradation of ATP by spinal roots is illustrated in Figure 5. At the beginning of the experiment, different concentrations of ATP were added to nerve-free bathing solutions until a final concentration of $165 \mathrm{nM}$ ATP was reached. Afterwards, apyrase (Figure $5 \mathrm{a}$ ) or four isolated spinal roots (Figure $5 \mathrm{~b}$ ) were added to the bathing solution. Both conditions produced a fast decline in the bath concentration of ATP. This indicates rapid nerve-dependent hydrolysis of ATP. In contrast, consumption of ATP by the light-producing enzymatic reaction is much slower in the range of ATP concentrations tested in these experiments.

The statistical analysis of differences in the kinetics of ATP degradation in cell-free culture dishes and in the presence of spinal roots is summarized in Figure 6. In all of the experiments with nerve segments, two pairs of spinal roots were tested. For the quantitative analysis, the photon counts obtained after addition of $150 \mathrm{nM}$ ATP were set to $100 \%$. Clearly, consumption of ATP by the luciferin/luciferase reaction is much slower as compared to ATP hydrolysis within the nerve trunk itself(Figure 6a). For example, $10 \mathrm{~min}$ after addition of ATP, a reduction of photon counts/s by $8.9 \pm$ $1.7 \%(n=6)$ was seen without nerve preparations whereas
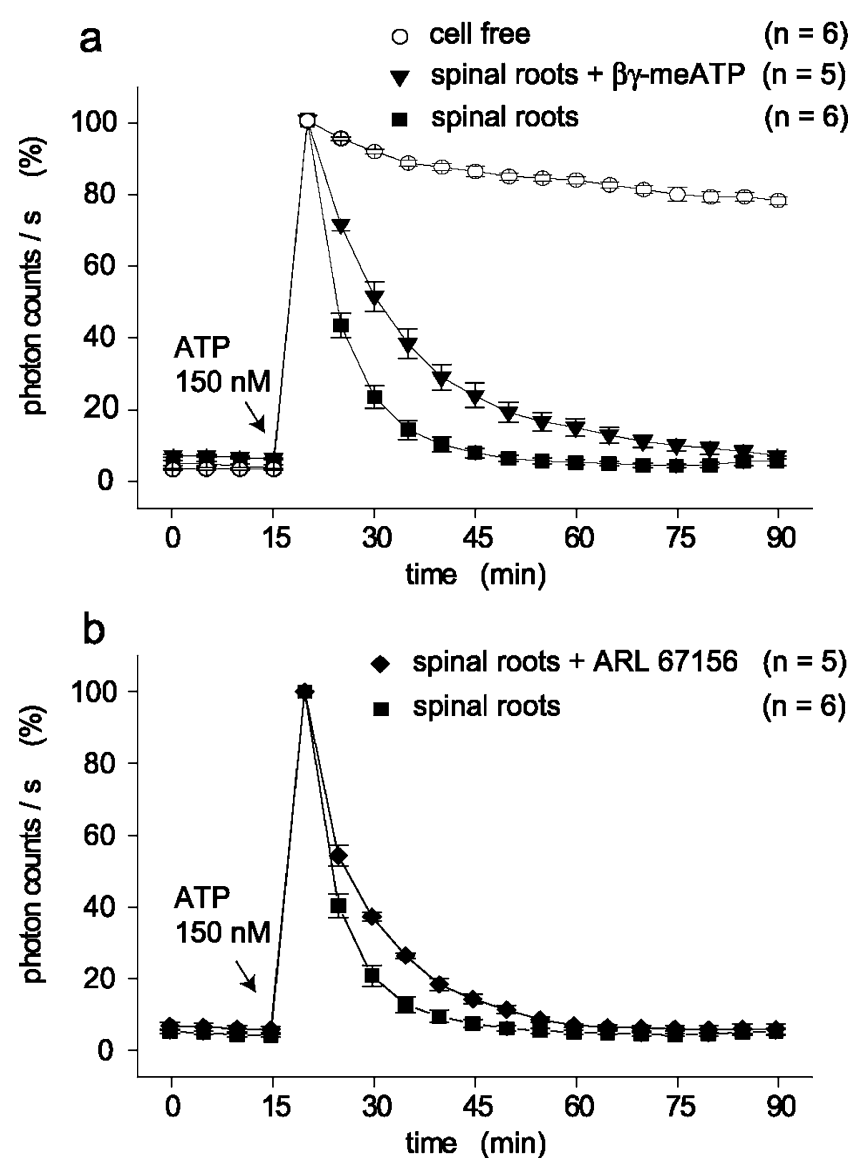

Figure 6 Modulation of ATP degradation by ecto-ATPase inhibitors. Kinetics of ATP degradation were tested in culture dishes which contained two pairs of spinal roots each. The concentration of ATP was measured every $5 \mathrm{~min}$ and is given in photon counts/s relative to the light intensity at $150 \mathrm{nM}$ ATP. This concentration of ATP was added $15 \mathrm{~min}$ after the start of the measurements and degradation of ATP was followed for a further $75 \mathrm{~min}$. (a) A comparison was made between cell-free culture dishes, spinal roots, and spinal roots in the presence of $\beta \gamma$-meATP $(300 \mu \mathrm{M})$. Degradation of ATP is much faster in the presence of spinal roots as compared to ATP consumption by a solution containing the ATP assay mix only; $\beta \gamma$-meATP had an inhibitory effect on ATP hydrolysis by spinal roots which is statistically significant (see text). (b) Kinetics of ATP degradation was tested after addition of ARL $67156(100 \mu \mathrm{M})$. Also this compound slightly, but significantly (see text), reduced hydrolysis of ATP seen in the presence of spinal roots 
a

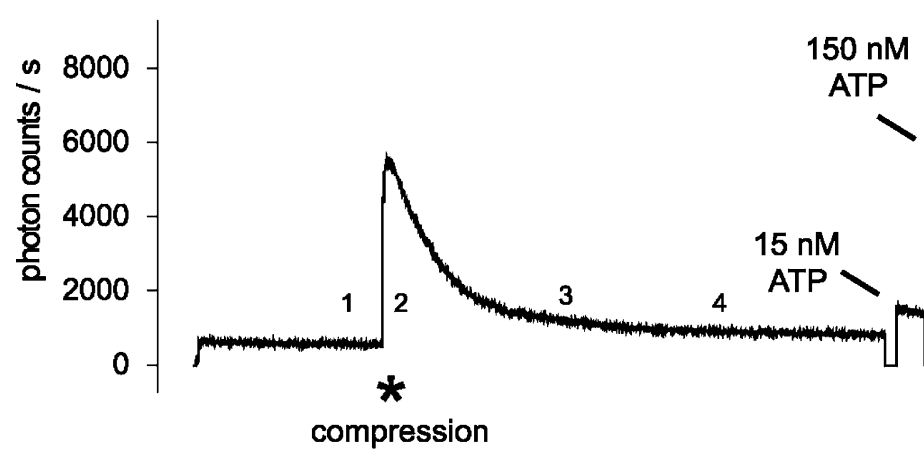

b

\section{A-fiber CAP}

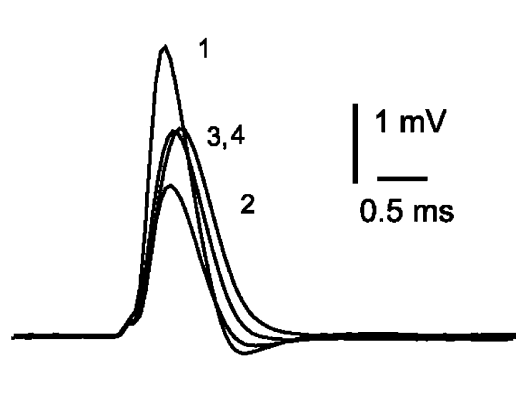

Figure 7 A standardized experimental protocol used for quantification of compression-induced ATP release. In these experiments, spinal roots were exposed to compression of $0.5 \mathrm{~mm}$ for $8 \mathrm{~s}$ (indicated by the asterisk in a). (a) Changes in the extracellular concentration of ATP were measured as an increase in light produced by the ATP assay mix and calibrated by means of two concentrations of ATP adjusted in the organ bath at the end of the experiment (the photomultiplier was shut

light intensity was reduced by $76.7 \pm 3.0 \%(n=6)$ in the presence of the spinal roots $(p<0.0001)$.

The contribution of ecto-nucleotidases to the degradation of ATP within the isolated rat spinal roots was tested by means of inhibitors of such enzymes (Figure 6a,b). Degradation of ATP was slower in the presence of either $\beta \gamma$-meATP $(300 \mu \mathrm{M})$ or ARL $67156(100 \mu \mathrm{M})$. However, when compared to the normal bath solution, either of the enzyme inhibitors only slightly reduced the ability of the spinal roots to metabolize ATP. On average, $10 \mathrm{~min}$ after addition of ATP, a reduction of photon counts/s by only $48.8 \pm 4.1 \%$ $(n=5)$ was seen in spinal roots in the presence of $\beta \gamma$ meATP as compared to $76.7 \pm 3.0 \%$ by control preparations $(p<0.001)$. The corresponding data for spinal roots in the presence of ARL 67156 are $62.9 \pm 1.2 \%(n=5, p<0.01)$.

\section{Kinetics of compression-induced ATP release}

The possible importance of ecto-nucleotidases for the decline of the ATP concentration after compression-induced release was tested in another series of experiments. Thereby, kinetics of ATP concentrations produced by transient compression of spinal roots were measured in control spinal roots and in spinal roots treated with $\beta \gamma$-meATP $(300 \mu \mathrm{M})$. This inhibitor was chosen because it produced stronger inhibition of ATP hydrolysis by spinal roots as compared to ARL 67156 (Figure 6). A typical example for these experiments is illustrated in Figure 7. After a resting period, the spinal root was transiently compressed by $0.5 \mathrm{~mm}$. This resulted in an increase of the extracellular ATP concentration and a reduction in the height of the A fiber compound action potential. The recovery from compression was followed for 30 min. Afterwards, two concentrations of ATP (15 and $150 \mathrm{nM}$ ) were adjusted in the organ bath for the conversion down during addition of ATP solutions; note discontinuity in the recording of bioluminescence). (b) Examples of compound A fiber potentials recorded at the points in time indicated with 1-4 in panel a. Note that nerve compression resulted in a decrease in peak amplitude which was largest during compression and recovered partially later during the recording (see also Figure 3)

of photon counts/s in concentrations of ATP (in nM). This standardized experimental protocol was applied to seven control spinal roots and seven spinal roots treated with $\beta \gamma$ meATP $(300 \mu \mathrm{M})$. Averaged data from these two groups of preparations were used to analyze extracellular concentrations of ATP reached (a) immediately after the mechanical stimulation (Figure $8 \mathrm{~b} ; t=0$ ) and (b) $4 \mathrm{~min}$ after the maximal ATP release (Figure $8 \mathrm{a}, \mathrm{b} ; t=240 \mathrm{~s}$ ). The mean compression-induced increase in the extracellular concentration of ATP in control spinal roots was $54.5 \pm 9.7 \mathrm{nM}(n=7)$. There was a larger rise in the presence of $\beta \gamma$-meATP $(88.9 \pm 16.1 \mathrm{nM}, n=7)$. However, this difference did not reach statistical significance. In contrast, $\beta \gamma$-meATP significantly reduced the kinetics of recovery measured either in percent or in absolute extracellular concentrations of ATP reached $4 \mathrm{~min}$ after nerve compression (Figure 8a, b; $t=240 \mathrm{~s}$ ).

\section{Discussion}

Compression and/or contusion of nerve trunks or spinal roots are common clinical situations with important consequences for nerve function. Previously, changes in electrophysiological parameters of myelinated axons produced by such mechanical stimuli have been studied in detail [2]. In white matter strips from the ventral spinal cord, it was found that nerve compression produces an immediate, partially reversible decrease of the A fiber compound action potential followed by a persistent conduction block in some of the myelinated axons. These changes in the electrophysiological parameters of A fibers have been also observed in the spinal roots used in the present study (Figures 3 and 7). The new, additional findings are effects of nerve compression on the concentration of ATP in the extracellular space of an isolated peripheral nerve trunk. Changes in the extracellular 

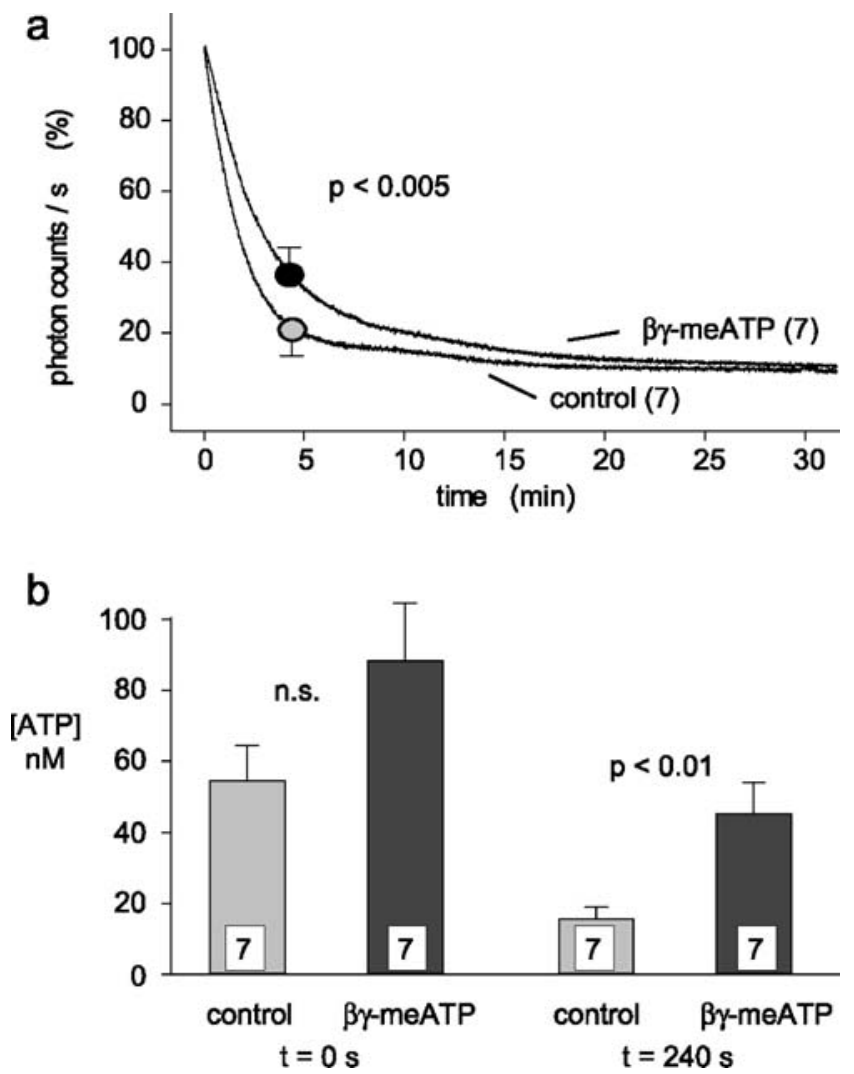

Figure 8 Effects of $\beta \gamma$-meATP on compression-induced release of ATP. Fourteen different isolated spinal roots were tested with the experimental protocol illustrated in Figure 7. (a) Averaged data of the decline in light production observed after compression of isolated spinal roots tested either in control solution or in the presence of $\beta \gamma$ meATP $(300 \mu \mathrm{M})$. The maximal light production (in photon counts/s) was set to $100 \%$ and data obtained after 4 min were used for statistical analysis. Note that $\beta \gamma$-meATP produces a small, but significant, slowing of the decay time. (b) Averaged data from the experiments illustrated in (a) are plotted as concentrations of ATP reached in the bath solution immediately after nerve compression $(t=0 \mathrm{~s})$ and $4 \mathrm{~min}$ later $(t=240 \mathrm{~s})$. Compression of spinal roots in the presence of $\beta \gamma$ meATP resulted in higher concentration of ATP and statistically significant slower recovery

concentration of ATP were measured with a time resolution of $1 \mathrm{~s}$. Such online recordings of ATP concentrations were possible because the ATP assay mix was added directly into the organ bath. As described before for epithelia [19], cultured astrocytes [20], and postnatal sciatic nerve [21], the mixture of saline and ATP assay mix was well tolerated by the isolated rat spinal roots used in the present study. The key observation is that nerve compression produces an elevation of the extracellular ATP concentration which returns to the baseline level within a few minutes only. It is very likely that cell lysis contributes to ATP release produced by nerve compression. However, to our knowledge, quantitative aspects and the time course of this type of ATP release are described for the first time. The findings indicate (a) that sufficient ATP is released for activation of axonal P2X receptors and (b) that rapid hydrolysis of ATP may produce enough adenosine for activation of axonal adenosine (A2) receptors. This indicates that chemosensitivity of axons to purines may contribute to the pathophysiology of painful carpal tunnel and other nerve entrapment syndromes.

To our knowledge, there is only one previous quantitative report about kinetics of ATP release related to contusion of nervous tissue: Wang et al. [24] studied the release of ATP following acute impact spinal cord injury. An ATP imaging technique was used to follow ATP release in the setting of this trauma. Imaging was initiated within 10 min after impact, and at this point in time the extracellular concentration of ATP was not elevated in the center of the injury. The authors conclude that ATP released on impact may be hydrolyzed within minutes; our data support this view. A further finding reported by Wang et al. [24] is a sustained process of pathologically high ATP release in the peritraumatic zone. We do not know whether a similar process takes place in spinal root segments remote from the region of compression because the field of view for the detection of bioluminescence was restricted to the lesion.

Our data reveal that compression injury of a peripheral nerve trunk produces high concentrations of extracellular ATP only for an initial transient period. One factor responsible for these fast kinetics may be the in vitro situation which allows rapid diffusion of ATP from the region of nerve compression into the surrounding bathing solution. However, it is also possible that the decline of the ATP concentration is due to extracellular enzymatic degradation and/or consumption by the ATP assay mix. The possible contribution of ATP hydrolysis to the degradation of ATP in isolated spinal roots was studied by means of known inhibitors of ecto-nucleotidases [13]. One of the compounds used, $\beta \gamma$-meATP, is a potent blocker of ATP hydrolysis by cultured astrocytes [20]. Also, ARL 67156 is often used as a blocker of ecto-ATPases [25]. However, application of either compound resulted only in a slight inhibition of ATP hydrolysis by isolated rat spinal roots (see Figure 6). Also in the isolated rabbit vagus nerve, an incomplete effect of $\beta \gamma$-meATP on ATP hydrolysis has been described [15]. The reason for these weak effects can be explained in the light of recent findings. It has been described that $\beta \gamma$-meATP preferentially targets the eNPP family of ecto-ATPases (eNPP1 and/or 3) over the CD39 family of eNTPDases [22]. However, the opposite situation, i.e., a prominent role of eNTPDases for hydrolysis of ATP, seems to be important for peripheral nerve preparations. In particular, the presence of eNTPDase2 was detected on nonmyelinating Schwann cells [16]. ARL 67156, too, only slightly inhibited ATP hydrolysis by isolated rat spinal roots. This observation is in accordance with the notion that this reagent acts as a modestly efficacious inhibitor of both eNPP and eNTPDase-type nucleotidases [22]. In a recent study, ARL 67156 revealed the highest inhibitory potency 
for NTPDase1. It was considerably less effective on NTPDase3 and essentially ineffective on NTPDase2 [26].

The possible contribution of ecto-nucleotidases for the kinetics of ATP concentration following nerve compression was tested by means of $\beta \gamma-$ meATP because this compound was more potent as a blocker of ecto-nucleotidases in spinal roots as compared to ARL 67156. It was observed that $\beta \gamma-$ meATP (a) enhanced the compression-induced release of ATP into the bath solution and (b) induced a slowing of the decline in the extracellular ATP concentration in the period following the compression (see Figure 8). Taken together, these observations indicate that an increase in the extracellular concentration of ATP in the trunk of a peripheral nerve is limited by activity of ecto-nucleotidases. However, at present, a more quantitative analysis is not possible due to the lack of potent inhibitors of such enzymes in peripheral nerve.

It is possible that ATP release produced by compression of a peripheral nerve trunk contributes to "positive" symptoms such as the development of pain. However, the concentrations of ATP reached in the extracellular space after nerve injury were only in the nanomolar range (see Figure 4). Such concentrations of ATP do not activate purinergic receptors in the membrane of nociceptive neurons. However, bioluminescence measured in the present study is mainly induced by ATP in the solution surrounding the nerve trunk. It is very likely that much higher concentrations of ATP are reached in the small extracellular space in the vicinity of nociceptive axons. It has been estimated that only $1 \%$ or less of the intracellular ATP pool needs to be released to activate maximally any and all purinoceptors [27]. In fact, cytosolic ATP released by cell damage produces transient currents in cultured sensory (nociceptive) neurons via activation of ionotropic P2X receptors [28]. This is an example for a fast functional response due to a transient elevation of extracellular ATP concentration. However, there are several possibilities of how short lasting extracellular ATP transients may induce long lasting alterations in axonal excitability and/or glial activity: (a) degradation of ATP to adenosine could result in an increase of axonal excitability in unmyelinated peripheral nerve fibers via metabotropic A2 receptors [7, 18]. Production of adenosine from ATP has been shown for isolated vagus nerves [15] and pain-enhancing actions of adenosine via P1 (A2) receptors have been described [5, 17]. (b) Long lasting activity in peripheral nociceptive neurons has been discussed as the result of cytokine release induced by activation of purinergic $\mathrm{P} 2 \mathrm{X}_{7}$ receptors [29, 30]. (c) Activation of $\mathrm{P} 2 \mathrm{Y}$ receptors on Schwann cells [9-12] may have long lasting consequences similar to the development of P2Y-related gliosis observed in astrocytes [31, 32].

Acknowledgments We would like to thank Ms. Christina Müller for valuable technical assistance and Dr. Uta Rundshagen for help in some of the experiments. This study was supported by the Deutsche Forschungsgemeinschaft (SFB 391/A1).

\section{References}

1. Stewart JD (1993) Compression and entrapment neuropathies. In: Dyck PJ, Thomas PK (eds) Peripheral neuropathy. Saunders, Philadelphia, pp 961-979

2. Shi R, Blight AR (1996) Compression injury of mammalian spinal cord in vitro and the dynamics of action potential conduction failure. J Neurophysiol 76:1572-1580

3. Maxwell WL, Irvine AG, Adams JH, Gennarelli TA, Tipperman R, Sturatis M (1991) Focal axonal injury: the early axonal response to stretch. J Neurocytol 20:157-164

4. Lazarowski ER, Boucher RC, Harden TK (2003) Mechanisms of release of nucleotides and integration of their action as $\mathrm{P} 2 \mathrm{X}$ - and P2Y-receptor activating molecules. Mol Pharmacol 64:785-795

5. Burnstock G, Wood JN (1996) Purinergic receptors: their role in nociception and primary afferent neurotransmission. Curr Opin Neurobiol 6:526-532

6. Chizh BA, Illes P (2001) P2X receptors and nociception. Pharmacol Rev 53:553-568

7. Irnich D, Tracey DJ, Polten J, Burgstahler R, Grafe P (2002) ATP stimulates peripheral axons in human, rat and mouse - differential involvement of $\mathrm{A}_{2 \mathrm{~B}}$ adenosine and $\mathrm{P} 2 \mathrm{X}$ purinergic receptors. Neuroscience 110:123-129

8. Labrakakis C, Tong CK, Weissman T, Torsney C, MacDermott $\mathrm{AB}$ (2003) Localization and function of ATP and $\mathrm{GABA}_{\mathrm{A}}$ receptors expressed by nociceptors and other postnatal sensory neurons in rat. J Physiol (Lond) 549:131-142

9. Lyons SA, Morell P, McCarthy KD (1994) Schwann cells exhibit P2Y purinergic receptors that regulate intracellular calcium and are up-regulated by cyclic AMP analogues. J Neurochem 63:552-560

10. Mayer C, Quasthoff S, Grafe P (1998) Differences in the sensitivity to purinergic stimulation of myelinating and nonmyelinating Schwann cells in peripheral human and rat nerve. Glia 23:374-382

11. Stevens B, Fields RD (2000) Response of Schwann cells to action potentials in development. Science 287:2267-2271

12. Weick M, Cherkas PS, Hartig W, Pannicke T, Uckermann O, Bringmann A, Tal M, Reichenbach A, Hanani M (2003) P2 receptors in satellite glial cells in trigeminal ganglia of mice. Neuroscience 120:969-977

13. Zimmermann H (2000) Extracellular metabolism of ATP and other nucleotides. Naunyn-Schmiedebergs Arch Pharmacol 362:299-309

14. Kukulski F, Levesque SA, Lavoie EG, Lecka J, Bigonnesse F, Knowles AF, Robson SC, Kirley TL, Sevigny J (2005) Comparative hydrolysis of $\mathrm{P} 2$ receptor agonists by NTPDases 1, 2, 3 and 8. Purinergic Signalling 1:193-204

15. Maire JC, Medilanski J, Straub RW (1984) Release of adenosine, inosine and hypoxanthine from rabbit non-myelinated nerve fibres at rest and during activity. J Physiol (Lond) 357:67-77

16. Braun N, Sévigny J, Robson SC, Hammer K, Hanani M, Zimmermann H (2004) Association of the ecto-ATPase NTPDase2 with glial cells of the peripheral nervous system. Glia 45:124-132

17. Sawynok J (1998) Adenosine receptor activation and nociception. Eur J Pharmacol 347:1-11

18. Lang PM, Tracey DJ, Irnich D, Sippel W, Grafe P (2002) Activation of adenosine and $\mathrm{P} 2 \mathrm{Y}$ receptors by ATP in human peripheral nerve. Naunyn-Schmiedebergs Arch Pharmacol 366:449-457

19. Taylor AL, Kudlow BA, Marrs KL, Gruenert DC, Guggino WB, Schwiebert EM (1998) Bioluminescence detection of ATP release mechanisms in epithelia. Am J Physiol 275:C1391-C1406

20. Joseph SM, Buchakjian MR, Dubyak GR (2003) Colocalization of ATP release sites and ecto-ATPase activity at the extracellular surface of human astrocytes. J Biol Chem 278:23331-23342

21. Liu GJ, Bennett MR (2003) ATP secretion from nerve trunks and Schwann cells mediated by glutamate. Neuroreport 14:2079-2083 
22. Joseph SM, Pifer MA, Przybylski RJ, Dubyak GR (2004) Methylene ATP analogs as modulators of extracellular ATP metabolism and accumulation. Br J Pharmacol 142:1002-1014

23. Ford SR, Chenault KH, Bunton LS, Hampton GJ, McCarthy J, Hall MS, Pangburn SJ, Buck LM, Leach FR (1996) Use of firefly luciferase for ATP measurement: other nucleotides enhance turnover. J Biolumin Chemilumin 11:149-167

24. Wang X, Arcuino G, Takano T, Lin J, Peng WG, Wan P, Li P, Xu Q, Liu QS, Goldman SA, Nedergaard M (2004) P2X7 receptor inhibition improves recovery after spinal cord injury. Nat Med 10:821-827

25. Crack BE, Pollard CE, Beukers MW, Roberts SM, Hunt SF, Ingall AH, McKechnie KC, IJzerman AP, Leff P (1995) Pharmacological and biochemical analysis of FPL 67156, a novel, selective inhibitor of ecto-ATPase. Br J Pharmacol 114:475-481

26. Iqbal J, Vollmayer P, Braun N, Zimmermann H, Müller CE (2005) A capillary electrophoresis method for the characterization of ecto-nucleoside triphosphate diphosphohydrolases (NTPDases) and the analysis of inhibitors by in-capillary enzymatic microreaction. Purinergic Signalling 1:349-358
27. Schwiebert EM, Zsembery A (2003) Extracellular ATP as a signaling molecule for epithelial cells. Biochem Biophys Acta 1615:7-32

28. Cook SP, McCleskey EW (2002) Cell damage excites nociceptors through release of cytosolic ATP. Pain 95:41-47

29. Colomar A, Marty V, Medina C, Combe C, Parnet P, Amedee T (2003) Maturation and release of interleukin-1beta by lipopolysaccharide-primed mouse Schwann cells require the stimulation of P2X7 receptors. J Biol Chem 278:30732-30740

30. Chessell IP, Hatcher JP, Bountra C, Michel AD, Hughes JP, Green P, Egerton J, Murfin M, Richardson J, Peck WL, Grahames CB, Casula MA, Yiangou Y, Birch R, Anand P, Buell GN (2005) Disruption of the P2X(7) purinoceptor gene abolishes chronic inflammatory and neuropathic pain. Pain 114:386-396

31. Franke H, Krugel U, Grosche J, Heine C, Hartig W, Allgaier C, Illes P (2004) P2Y receptor expression on astrocytes in the nucleus accumbens of rats. Neuroscience 127:431-441

32. Neary JT, Kang Y, Willoughby KA, Ellis EF (2003) Activation of extracellular signal-regulated kinase by stretch-induced injury in astrocytes involves extracellular ATP and P2 purinergic receptors. J Neurosci 23:2348-2356 